\title{
An Immersed Finite Element Space and Its Approximation Capability *ł⿳亠丷厂
}

\author{
Z. Li $\$$ T. Lin, Y. Lin, R.C. Rogers**
}

\begin{abstract}
An immersed finite element (IFE) space is introduced for interface problems of second order elliptic partial differential equations. The IFE space is nonconforming and its partition can be indenpendent of the interface. The error estimates for the interpolation of a function in the usual Sobolev space indicates that the IFE space has an approximation capability similar to that of the standard linear finite element space except for a logarithm factor.
\end{abstract}

\section{Introduction}

In this paper, we will discuss the approximation capability of the immersed finite element (IFE) space formed by first degree polynomials for the following interface problem:

$$
\begin{aligned}
& -\nabla \cdot(\beta \nabla u)=f, \quad(x, y) \in \Omega, \\
& \left.u\right|_{\partial \Omega}=g,
\end{aligned}
$$

together with the jump conditions on the interface $\Gamma$ :

$$
\begin{aligned}
{\left.[u]\right|_{\Gamma} } & =0 \\
{\left.\left[\beta u_{n}\right]\right|_{\Gamma} } & =0 .
\end{aligned}
$$

Here, see the sketch in Figure $1, \Omega \subset R^{2}$ is a convex polygonal domain, the interface $\Gamma$ is a curve separating $\Omega$ into two domains $\Omega^{-}, \Omega^{+}$such that $\Omega=\Omega^{-} \cup \Omega^{+}$, and the coefficient $\beta(x, y)$ is a piecewise constant function defined by

$$
\beta(x, y)= \begin{cases}\beta^{-}, & (x, y) \in \Omega^{-}, \\ \beta^{+}, & (x, y) \in \Omega^{+} .\end{cases}
$$

The basic idea of the immerse finite elements is to form the partition $\mathcal{T}_{h}$ independent of interface $\Gamma$ so that partitions with simple and efficient structures, such as a Cartesian partition,

\footnotetext{
${ }^{*} \mathrm{MSC}(2000)$ Primary: 65N15, 65N30, 65N50, 35R05.

${ }^{\dagger}$ Key words: interface problems, immersed interface, finite element, error estimates.

${ }^{\ddagger}$ This work is supported partially by the NSF grant DMS-9704621, DMS-0073403, the Army grant ARO-39676MA, the NSERC (Canada).

${ }^{\S}$ Center for Research in Scientific Computation \& Department of Mathematics, North Carolina State University, Raleigh, NC 27695-8205

`Department of Mathematics, Virginia Tech, Blacksburg, VA 24061, tlin@math.vt.edu

"Department of Mathematical Sciences, University of Alberta, Edmonton, Alberta, Canada T6G 2G1

${ }^{* *}$ Department of Mathematics, Virginia Tech, Blacksburg, VA 24061
} 


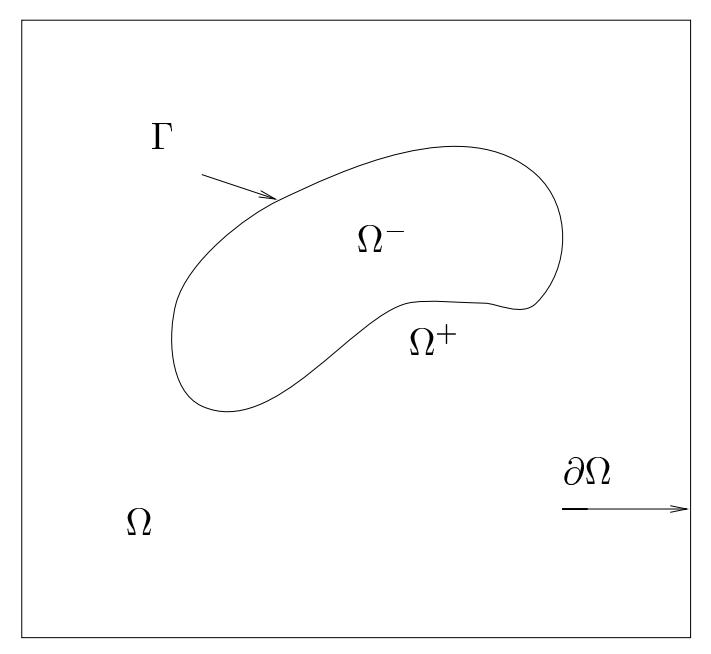

Figure 1: A sketch of the domain for the interface problem.

can be used to solve an interface problem with a rather complicate or varying interface. We only consider partitions formed by triangles here, partitions formed by quadrilateral elements will be discussed in a forthcoming paper. Without loss of generality, we assume that the triangles in the partition used have the following features:

$\left(H_{1}\right)$ : If $\Gamma$ meets one edge of a triangle at more than two points, then this edge is part of $\Gamma$.

$\left(H_{2}\right)$ : If $\Gamma$ meets a triangle at two points, then these two points must be on different edges of this triangle.

Obviously, triangles in a partition can be separated into two classes:

- Non-interface triangle: The interface $\Gamma$ either does not intersect with this triangle, or it intersects with this triangle but does not separate its interior into two non-trivial subsets.

- Interface triangle: The interface $\Gamma$ cuts through its interior.

In a non-interface triangle, the local nodal basis functions will be the standard linear polynomials, but in an interface triangle, the local nodal basis functions will be formed by piecewise linear polynomials that can satisfy the interface conditions (1.3) and (1.4) in a sense of approximation explained in the next section. The immersed finite element space defined over the whole domain $\Omega$ with the partition chosen can then be defined in the usual way.

This report is organized as follows. In Section 2, we will introduce the IFE space and describe some basic properties of the local nodal basis functions of the IFE space. In Section 3, we will use the technique based on the multipoint Taylor expansion, see [2,3], to derive error estimates for the interpolation in the IFE space of the functions in Sobolev spaces. We would also like to point out that several arguments used in the error estimation are inspired by [4].

For any subset $T$ of $\Omega$, we let

$$
T^{s}=T \cap \Omega^{s}, \quad s=-,+.
$$

For any function $f(x, y)$ defined in $T \subset \Omega$, we can restrict it to $T^{s}, s=-,+$ to obtain two functions as

$$
f^{s}(x, y)=f(x, y), \text { if }(x, y) \in T^{s}, s=-,+.
$$

We use $\overline{D E}$ to denote the line segment between two points $D, E \in \Omega$. For any curve $\Gamma$, we use $\mathbf{n}_{\Gamma}$ to denote its unit normal vector pointing to a particular side of $\Gamma$. Also, for any measurable subset $\Lambda$ of $\Omega$, we use $|\Lambda|$ to denote its measure. 


\section{The immersed finite element space}

In this section, we will first introduce the local nodal IFE basis functions and then use them to define the IFE space over the whole domain with a partition chosen. We will also describe some basic features of these basis functions.

For a typical triangle $T \in \mathcal{T}_{h}$, we use $A=\left(x_{1}, y_{2}\right)^{T}, B=\left(x_{2}, y_{2}\right)^{T}, C=\left(x_{3}, y_{3}\right)^{T}$ to denote its vertices, and use $D=\left(x_{D}, y_{D}\right)^{T}$ and $E=\left(x_{E}, y_{E}\right)^{T}$ to denote its interface points on the edges if $T$ is an interface triangle ( see the sketch in Figure 2).

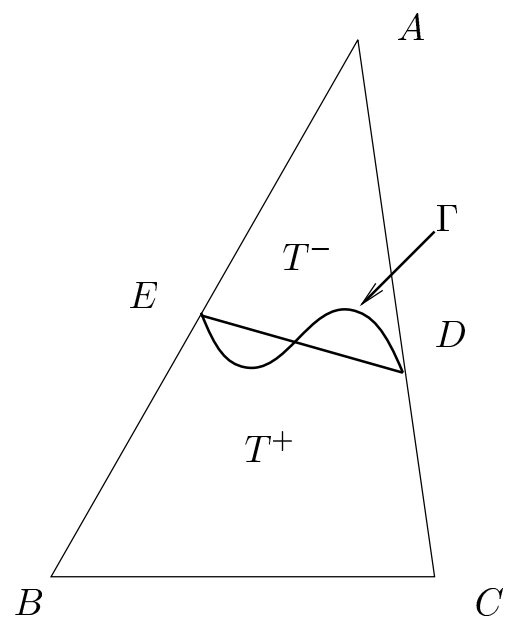

Figure 2: A typical interface triangle $\triangle A B C$. The curve between $D$ and $E$ is part of the interface $\Gamma$.

Our main concern is the finite element functions in an interface triangle. We follow an idea similar to that for the Hsieh-Clough-Tocher macro $C^{1}$ element [1] in which piecewise polynomials are used in a triangle to maintain certain desirable features. For our interface problem, we obviously would like the finite element functions to satisfy the jump conditions across the interface. Since the interface $\Gamma$ separates an interface triangle $T$ into two subsets $T^{-}$and $T^{+}$, we naturally can try to form a finite element function by two first degree polynomials defined in $T^{-}$ and $T^{+}$, respectively. Note that each polynomial of degree one has three freedoms (coefficients). The values of the finite element function at the vertices of $T$ provides three restrictions. The normal derivative jump condition provides another. Then we can have two more restrictions by requiring the continuity of the finite element function at interface points $D$ and $E$. Intuitively, these six conditions can yield the desired piecewise linear polynomial in an interface triangle. This leads us to consider functions defined as follows:

$$
\phi(x, y)= \begin{cases}\phi^{-}(x, y)=a_{1} x+b_{1} y+c_{1}, & (x, y) \in T^{-} \\ \phi^{+}(x, y)=a_{2} x+b_{2} y+c_{2}, & (x, y), \in T^{+} \\ \phi^{-}(D)=\phi^{+}(D), \phi^{-}(E)=\phi^{+}(E), & \\ \left(\beta^{-} \nabla \phi^{-}-\beta^{+} \nabla \phi^{+}\right) \cdot \mathbf{n}(\overline{D E})=0, & \end{cases}
$$

where $\mathbf{n}(\overline{D E})$ is the unit vector perpendicular to the line $\overline{D E}$.

As usual, we only need to define the nodal IFE basis functions in the reference triangle $\hat{T}$ with vertices $\hat{A}, \hat{B}$ and $\hat{C}$ :

$$
\hat{A}=\left(\begin{array}{l}
0 \\
1
\end{array}\right), \hat{B}=\left(\begin{array}{l}
0 \\
0
\end{array}\right), \hat{C}=\left(\begin{array}{l}
1 \\
0
\end{array}\right) .
$$


The interface triangle $T$ is related with the reference triangle by the usual affine mapping:

$$
F(\hat{\mathbf{x}})=\mathbf{x}_{B}+M \hat{\mathbf{x}}
$$

Under this mapping, $D$ becomes $\hat{D}=\left(0, \hat{y}_{1}\right)^{T}, E$ become $\hat{E}=\left(1-\hat{y}_{2}, \hat{y}_{2}\right)^{T}$ with $0<\hat{y}_{1}, \hat{y}_{2}<1$, and

$$
\phi(X)=\hat{\phi}\left(F^{-1}(X)\right)=\hat{\phi}(\hat{X})
$$

In the reference triangle $\hat{T}$, the basis function $\hat{\phi}$ has the following format:

$$
\hat{\phi}(\hat{X})= \begin{cases}\hat{\phi}^{+}(\hat{X})=\hat{\phi}^{+}(\hat{A})+a_{1} \hat{x}+a_{2}(\hat{y}-1), & \hat{X} \in \hat{T}^{+}, \\ \hat{\phi}^{-}(\hat{X})=\hat{\phi}(\hat{B})+(\hat{\phi}(\hat{C})-\hat{\phi}(\hat{B})) \hat{x}+b_{2} \hat{y}, & \hat{X} \in \hat{T}^{-} .\end{cases}
$$

The continuity of $\hat{\phi}$ at $\hat{D}$ and $\hat{E}$ lead to

$$
\begin{aligned}
a_{2}\left(\hat{y}_{1}-1\right)-b_{2} \hat{y}_{1} & =\hat{\phi}(\hat{B})-\hat{\phi}(\hat{A}), \\
a_{1}\left(1-\hat{y}_{2}\right)+a_{2}\left(\hat{y}_{2}-1\right)-b_{2} \hat{y}_{2} & =\hat{\phi}(\hat{B})-\hat{\phi}(\hat{A})+(\hat{\phi}(\hat{C})-\hat{\phi}(\hat{B}))\left(1-\hat{y}_{2}\right) .
\end{aligned}
$$

The flux jump condition $\left(\beta^{-} \nabla \phi^{-}-\beta^{+} \phi^{+}\right) \cdot \mathbf{n}(\overline{D E})=0$ becomes

$$
\left(\beta^{-} \nabla \hat{\phi}^{-}-\beta^{+} \hat{\phi}^{+}\right) \cdot \hat{\mathbf{n}}(\overline{D E})=0, \text { with } \hat{\mathbf{n}}(\overline{D E})=M^{-1} \mathbf{n}(\overline{D E})=\left(\begin{array}{c}
\hat{n}_{1} \\
\hat{n}_{2}
\end{array}\right),
$$

which leads to

$$
a_{1} \hat{n}_{1}+a_{2} \hat{n}_{2}-\rho \hat{n}_{2} b_{2}=\rho\left(\hat{\phi}_{i}(\hat{C})-\hat{\phi}_{i}(\hat{B})\right) \hat{n}_{1}
$$

with $\rho=\beta^{-} / \beta^{+}$. Assuming that $\hat{n}_{2} \neq 0$, we can write the above as

$$
a_{1} \hat{\alpha}-a_{2}+\rho b_{2}=\rho\left(\hat{\phi}_{i}(\hat{C})-\hat{\phi}_{i}(\hat{B})\right) \hat{\alpha}, \quad \text { with } \hat{\alpha}=-\frac{\hat{n}_{1}}{\hat{n}_{2}} .
$$

Lemma 2.1 The function $\phi(x, y)$ defined by (2.5) in an interface triangle $T$ is uniquely decided by its values at the three vertices of $T$.

Proof. We only carry out the proof for $\hat{\phi}$. Equations (2.8), (2.8), and (2.10) for a linear system about $a_{1}, a_{2}$ and $b_{2}$ whose matrix is

$$
\mathcal{A}=\left(\begin{array}{ccc}
0 & \hat{y}_{1}-1 & -\hat{y}_{1} \\
1-\hat{y}_{2} & \hat{y}_{2}-1 & \hat{y}_{2} \\
\hat{\alpha} & -1 & \rho
\end{array}\right)
$$

and the right hand side of this system is

$$
\mathbf{r}=\left(\begin{array}{c}
\hat{\phi}_{i}(\hat{B})-\hat{\phi}_{i}(\hat{A}) \\
\hat{\phi}_{i}(\hat{B})-\hat{\phi}_{i}(\hat{A})+\left(\hat{\phi}_{i}(\hat{C})-\hat{\phi}_{i}(\hat{B})\right)\left(1-\hat{y}_{2}\right) \\
\rho\left(\hat{\phi}_{i}(\hat{C})-\hat{\phi}_{i}(\hat{B})\right) \hat{\alpha}
\end{array}\right) .
$$

Assuming that the line passing $\hat{D}$ and $\hat{E}$ is in the direction $(1, \alpha)^{T}$, then

$$
\alpha=\frac{\hat{y}_{2}-\hat{y}_{1}}{1-\hat{y}_{2}}, \quad \hat{y}_{2} \neq 1
$$


and

$$
\begin{aligned}
\operatorname{det}(\mathcal{A}) & =\left(1-\hat{y}_{2}\right)\left(\hat{y}_{1}+\hat{\alpha} \alpha+\rho\left(1-\hat{y}_{2}\right)(1+\alpha)\right) \\
& =\left(1-\hat{y}_{2}\right)\left(\hat{y}_{1}+\hat{\alpha} \alpha+\rho\left(1-\frac{\hat{y}_{1}+\alpha}{1+\alpha}\right)(1+\alpha)\right) \\
& =\left(1-\hat{y}_{2}\right)\left(\hat{y}_{1}+\hat{\alpha} \alpha+\rho\left(1-\hat{y}_{1}\right)\right) \\
& \geq\left(1-\hat{y}_{2}\right)(\hat{\alpha} \alpha+\min \{1, \rho\})>0
\end{aligned}
$$

because $0 \leq \hat{y}_{1} \leq 1$. This implies that this linear system has a unique solution, and the function $\hat{\phi}$ or $\phi$ is uniquely determined by the jump conditions and its values at vertices of the triangle.

Now, we let $\phi_{i}(X)$ be the piecewise linear function described by $(2.5)$ and

$$
\phi_{i}\left(x_{j}, y_{j}\right)= \begin{cases}1, & \text { if } i=j, \\ 0, & \text { if } i \neq j,\end{cases}
$$

for $1 \leq i, j \leq 3$. Moreover, we now let $S_{h}(T)$ be the linear space of all the functions defined by (2.5), and call it the immersed finite element space on an interface triangle $T$. Furthermore, we notice:

- The proof of Lemma 2.1 itself provides a way to construct the nodal basis functions in an interface triangle, and $\phi_{i}(X), i=1,2,3$ form a basis of $S_{h}(T)$.

- ¿From the proof we can see that $\phi^{-}(x, y)=\phi^{+}(x, y)$ when $\rho=1$, i.e., when the coefficient does not have the jump, the functions in $S_{h}(T)$ become the usual linear polynomials. In this case, $S_{h}(T)$ reduces to the standard linear finite element space.

- When $\Gamma \cap T$ is a straight line, the function $\phi(x, y)$ defined by $(2.5)$ is continuous in $T$ and therefore is in $H^{1}(T)$.

We now turn to the discussion on the properties of the IFE functions.

Lemma 2.2 For an interface triangle $T$, every function $\phi \in S_{h}(T)$ satisfies the flux jump condition on $\Gamma \cap T$ exactly in the following weak sense:

$$
\int_{\Gamma \cap T}\left(\beta^{-} \nabla \phi^{-}-\beta^{+} \nabla \phi^{+}\right) \cdot \mathbf{n}_{\Gamma} d s=0 .
$$

Proof. For any $\phi \in S_{h}(T)$, it is obvious that $\phi^{s} \in H^{2}\left(T^{s}\right), \quad s=-,+$. Also, because $\phi$ is a piecewise linear polynomial satisfying (2.5), Green's formula leads to

$$
\int_{\Gamma \cap T}\left(\beta^{-} \nabla \phi^{-}-\beta^{+} \nabla \phi^{+}\right) \cdot \mathbf{n}_{\Gamma} d s=-\int_{\overline{D E}}\left(\beta^{-} \nabla \phi^{-}-\beta^{+} \nabla \phi^{+}\right) \cdot \mathbf{n}_{\overline{D E}} d s=0 .
$$

Lemma 2.3 For the three functions $\phi_{i}(X), i=1,2,3$ defined above, we have

$$
\phi_{1}(X)+\phi_{2}(X)+\phi_{3}(X)=1 .
$$

Proof. Again, we only need to show that this is true for $\hat{\phi}_{i}$ whose parameters in (2.7) are $a_{1 i}, a_{2 i}, b_{2 i}, i=1,2,3$. Using the linear system determining the parameters $a_{1}, a_{2}, b_{2}$ in the 
proof of the Lemma 2.1, we have

$$
\begin{aligned}
& a_{1 i}= \begin{cases}\frac{\alpha(1-\rho)}{\hat{y}_{1}+\hat{\alpha} \alpha+\rho\left(1-\hat{y}_{1}\right)}, & i=1, \\
\frac{-(1+\hat{\alpha} \alpha) \rho+(1+\alpha)(\rho-1) \hat{y}_{2}}{\hat{y}_{1}+\hat{\alpha} \alpha+\rho\left(1-\hat{y}_{1}\right)}, & i=2, \\
\frac{\rho+\alpha(-1+\rho+\hat{\alpha} \rho)-(1+\alpha)(\rho-1) \hat{y}_{2}}{\hat{y}_{1}+\hat{\alpha} \alpha+\rho\left(1-\hat{y}_{1}\right)}, & i=3,\end{cases} \\
& a_{2 i}= \begin{cases}\frac{\hat{\alpha} \alpha+\rho}{\hat{y}_{1}+\hat{\alpha} \alpha+\rho\left(1-\hat{y}_{1}\right)}, & i=1, \\
\frac{-(1+\hat{\alpha} \alpha) \rho+\hat{\alpha}(1+\alpha)(\rho-1) \hat{y}_{2}}{\hat{y}_{1}+\hat{\alpha} \alpha+\rho\left(1-\hat{y}_{1}\right)}, & i=2, \\
-\frac{\hat{\alpha}(-1+\rho)\left(-\alpha+(1+\alpha) \hat{y}_{2}\right)}{\hat{y}_{1}+\hat{\alpha} \alpha+\rho\left(1-\hat{y}_{1}\right)}, & i=3, \\
b_{2 i}= & \begin{cases}\frac{\hat{\alpha} \alpha+1}{\hat{y}_{1}+\hat{\alpha} \alpha+\rho\left(1-\hat{y}_{1}\right)}, & i=1, \\
\frac{-1-\hat{\alpha}(-1+\rho+\alpha \rho)+\hat{\alpha}(1+\alpha)(\rho-1) \hat{y}_{2}}{\hat{y}}, & i=2, \hat{\alpha} \alpha+\rho\left(1-\hat{y}_{1}\right)\end{cases} \\
-\frac{\hat{\alpha}(1+\alpha)(-1+\rho)\left(-1+\hat{y}_{2}\right)}{\hat{y}_{1}+\hat{\alpha} \alpha+\rho\left(1-\hat{y}_{1}\right)}, & i=3 .\end{cases}
\end{aligned}
$$

A simple calculation can show that

$$
\begin{aligned}
& a_{j 1}+a_{j 2}+a_{j 3}=0, j=1,2, \\
& b_{21}+b_{22}+b_{23}=0,
\end{aligned}
$$

which together with (2.7) lead to the result of this lemma.

In the discussion below, we need another assumption on the partition $\mathcal{T}_{h}$.

$\left(H_{3}\right)$ : The family of partitions $\mathcal{T}_{h}$ with $h>0$ is regular, see [7].

Lemma 2.4 There exists a constant $C$ such that for any interface triangle $T \in \mathcal{T}_{h}$ and $X \in T$ we have

$$
\begin{aligned}
& \left|\phi_{i}(X)\right| \leq C, \\
& \left\|\nabla \phi_{i}(X)\right\| \leq C h^{-1} .
\end{aligned}
$$

Proof. Obviously, (2.12) follows from the boundedness of the parameters $a_{1}, a_{2}, b_{2}$ in (2.7). ¿From the proof of the previous lemma, we can see that these coefficients are linear combinations of the following functions

$$
\frac{1}{\hat{y}_{1}+\hat{\alpha} \alpha+\rho\left(1-\hat{y}_{1}\right)}, \quad \frac{\alpha}{\hat{y}_{1}+\hat{\alpha} \alpha+\rho\left(1-\hat{y}_{1}\right)}, \quad \frac{\hat{\alpha} \alpha}{\hat{y}_{1}+\hat{\alpha} \alpha+\rho\left(1-\hat{y}_{1}\right)} .
$$

Without loss of generality, we can assume that $\alpha \geq 0$, and when $\alpha$ tends to $\infty$, $\hat{\alpha}$ will tend to $\hat{\alpha}_{\infty}$ by which $\left(\hat{\alpha}_{\infty},-1\right)^{T}$ describes the direction for the image of the normal vector of $\overline{B A}$ under the affine mapping $F(\hat{\mathbf{x}})$. Since the partition is regular, there must exist a constant $C$ 
such that $\hat{\alpha}_{\infty} \geq C>0$. Therefore all the functions listed above can be uniformly bounded by a constant for $\hat{\alpha}, \alpha \in[0, \infty]$. This implies the boundedness of $\hat{\phi}_{i}$, and then the boundedness of $\phi_{i}, i=1,2,3$.

Note that

$$
\nabla \hat{\phi}_{i}(\hat{X})= \begin{cases}\left(\begin{array}{l}
a_{1} \\
a_{2}
\end{array}\right), & \hat{X} \in \hat{T}^{+} \\
\left(\begin{array}{c}
\hat{\phi}_{i}(\hat{C})-\hat{\phi}_{i}(\hat{B}) \\
b_{2}
\end{array}\right), & \hat{X} \in \hat{T}^{-},\end{cases}
$$

and $\nabla \phi_{i}=M^{-T} \nabla \hat{\phi}_{i}$. Because of partition is regular, we have $\left\|M^{-T}\right\| \leq C h^{-1}$. Since $\left\|\nabla \hat{\phi}_{i}\right\|$ is bounded, we finally have

$$
\left\|\nabla \phi_{i}\right\| \leq C h^{-1}
$$

Now we use the partition $\mathcal{T}_{h}$ to define an immersed finite element (IFE) space $S_{h}(\Omega)$. We first define a nodal basis function $\phi(x, y)$ for each node $\left(x_{N}, y_{N}\right)^{t}$ of $\mathcal{T}_{h}$ piecewisely such that $\phi\left(x_{N}, y_{N}\right)=1$ but zero at other nodes, and $\left.\phi\right|_{T} \in S_{h}(T)$ for any triangle $T \in \mathcal{T}_{h}$. Here $S_{h}(T)$ is the usual space of linear polynomials when $T$ is a non-interface triangle, or the immersed finite element space on $T$ introduced above when $T$ is an interface triangle. Then we define $S_{h}(\Omega)$ as the span of these nodal basis functions, and it is easy to see that $S_{h}(\Omega)$ has the following properties:

- For a partition $\mathcal{T}_{h}$, the IFE space $S_{h}(\Omega)$ has the same number of nodal basis functions as that formed by the usual linear polynomials.

- For a partition $\mathcal{T}_{h}$ fine enough, most of its triangles are non-interface triangles, and most of the nodal basis functions of the IFE space $S_{h}(\Omega)$ are just the usual linear nodal basis functions except for few nodes in the vicinity of the interface $\Gamma$.

- For any $\phi \in S_{h}(\Omega)$, we have

$$
\left.\phi\right|_{\Omega \backslash \Omega^{\prime}} \in H^{1}\left(\Omega \backslash \Omega^{\prime}\right),
$$

where $\Omega^{\prime}$ is the union of interface triangles.

\section{Some error estimates for interpolation approximations}

For any $T \subset \Omega$, we let

$$
\begin{aligned}
& P W_{p}^{m}(T)=\left\{u|u|_{T^{s}} \in W_{p}^{m}\left(T^{s}\right), s=-,+\right\}, p \geq 1, m=0,1,2, \\
& P H_{i n t}^{2}(T)=\left\{u \in C(T),\left.u\right|_{T^{s}} \in H^{2}\left(T^{s}\right), s=-,+,\left[\beta \frac{\partial u}{\partial \mathbf{n}_{\Gamma}}\right]=0 \text { on } \Gamma \cap T\right\}, \\
& P C_{i n t}^{m}(T)=\left\{u \in C(T),\left.u\right|_{T^{s}} \in C^{m}\left(T^{s}\right), s=-,+\left[\beta \frac{\partial u}{\partial \mathbf{n}}\right]=0 \text { on } \Gamma\right\}, m \geq 2 .
\end{aligned}
$$

As usual, we have $P H^{m}(T)=P W_{2}^{m}(T)$. Obviously, we have $P H_{i n t}^{2}(T) \subset P H^{2}(T)$. Also, for any function $u \in P W_{p}^{m}(T)$, we let

$$
\|u\|_{m, p, T}^{2}=\|u\|_{m, p, T^{-}}^{2}+\|u\|_{m, p, T^{+}}^{2} .
$$


Semi-norms of $P W_{p}^{m}(T)$ can be defined accordingly by

$$
|u|_{m, p, T}^{2}=|u|_{m, T^{-}}^{2}+|u|_{m, p, T^{+}}^{2} .
$$

When $p=2$, we will drop $p$ from the notation of the norms. Similar definitions can be introduced for $P H^{m}(\Omega), P H_{\text {int }}^{2}(\Omega)$ and $P C^{3}(\Omega)$.

In this section, we assume that the interface curve $\Gamma$ and the partition $\mathcal{T}_{h}$ chosen satisfy the following assumptions:

$\left(H_{4}\right)$ : The interface curve $\Gamma$ is defined by a piecewise $C^{2}$ function, and the partition $\mathcal{T}_{h}$ is formed such that the subset of $\Gamma$ in any interface triangle is $C^{2}$.

$\left(H_{5}\right)$ : The interface $\Gamma$ is smooth enough such that $P C^{3}(T)$ is dense in $P H^{2}(T)$ for any interface triangle of $\mathcal{T}_{h}$.

Based on the regularity of the transmission problem, see $[6,9],\left(H_{5}\right)$ will be true if $\Gamma$ is smooth enough.

For a function $u \in P H_{i n t}^{2}(T), T \in \mathcal{T}_{h}$, we let $I_{h, T} u \in S_{h}(T)$ be its interpolation such that $I_{h, T} u(X)=u(X)$ when $X$ is a vertex of $T$. For an interface triangle $T$ with vertice $A, B, C$, we have

$$
I_{h, T} u(X)=u(A) \phi_{1}(X)+u(B) \phi_{2}(X)+u(C) \phi_{3}(X) .
$$

Accordingly, for a function $u \in P H_{\text {int }}^{2}(\Omega)$, we let $I_{h} u \in S_{h}(\Omega)$ be its interpolation such that $\left.I_{h} u\right|_{T}=I_{h, T}\left(\left.u\right|_{T}\right)$ for any $T \in \mathcal{T}_{h}$. The purpose of this section is to derive error estimates of the interpolation of $u \in P H_{i n t}^{2}(\Omega)$.

Since the error estimates in any non-interface triangle is well known, we focus our discussion on interface triangles. For an arbitrary interface triangle, see Figure 3, we let $T^{*}$ be the subset in $T$ enclosed by the interface $\Gamma$ and the line segment $\overline{D E}$, and let

$$
T^{* s}=T^{s} \backslash T^{*}, \quad s=-,+,
$$

For any point $\widetilde{A} \in \Gamma$, we let $\widetilde{A}_{\perp}$ be intersection point of the line segment $\overline{D E}$ and the line perpendicularly passing $\overline{D E}$ and $\widetilde{A}$. We will use the following four matrices:

$$
\begin{aligned}
N^{-}(\widetilde{A}) & =\left(\begin{array}{cc}
n_{y}(\widetilde{A})^{2}+\rho n_{x}(\widetilde{A})^{2} & (\rho-1) n_{x}(\widetilde{A}) n_{y}(\widetilde{A}) \\
(\rho-1) n_{x}(\widetilde{A}) n_{y}(\widetilde{A}) & n_{x}(\widetilde{A})^{2}+\rho n_{y}(\widetilde{A})^{2}
\end{array}\right), \\
N^{+}(\widetilde{A}) & =\left(\begin{array}{cc}
n_{y}(\widetilde{A})^{2}+\tilde{\rho} n_{x}(\widetilde{A})^{2} & (\tilde{\rho}-1) n_{x}(\widetilde{A}) n_{y}(\widetilde{A}) \\
(\tilde{\rho}-1) n_{x}(\widetilde{A}) n_{y}(\widetilde{A}) & n_{x}(\widetilde{A})^{2}+\tilde{\rho} n_{y}(\widetilde{A})^{2}
\end{array}\right), \quad \tilde{\rho}=\frac{1}{\rho}, \\
N_{\overline{D E}}^{-} & =\left(\begin{array}{cc}
\bar{n}_{y}^{2}+\rho \bar{n}_{x}^{2} & (\rho-1) \bar{n}_{x} \bar{n}_{y} \\
(\rho-1) \bar{n}_{x} \bar{n}_{y} & \bar{n}_{x}^{2}+\rho \bar{n}_{y}^{2}
\end{array}\right), \\
N_{\overline{D E}}^{+} & =\left(\begin{array}{cc}
\bar{n}_{y}^{2}+\tilde{\rho} \bar{n}_{x}^{2} & (\tilde{\rho}-1) \bar{n}_{x} \bar{n}_{y} \\
(\tilde{\rho}-1) \bar{n}_{x} \bar{n}_{y} & \bar{n}_{x}^{2}+\tilde{\rho} \bar{n}_{y}^{2}
\end{array}\right),
\end{aligned}
$$

where $\mathbf{n}(\widetilde{A})=\left(n_{x}(\widetilde{A}), n_{y}(\widetilde{A})\right)^{T}$ is the unit normal vector of $\Gamma$ at $\widetilde{A}$, and $\mathbf{n}(\overline{D E})=\left(\bar{n}_{x}, \bar{n}_{y}\right)^{T}$ is the unit normal vector of $\overline{D E}$.

Since $\Gamma \cap T$ is a $C^{2}$ curve, when the partition $\mathcal{T}_{h}$ is fine enough, we can introduce a local coordinate system centered at point $D$ with one axis in the direction of $\overline{D E}$. For any point $(x, y)^{T}$, let $(\xi, \eta)$ be its coordinates in this local coordinate system. Then we have

$$
\left(\begin{array}{l}
x \\
y
\end{array}\right)=\left(\begin{array}{c}
x_{D} \\
y_{D}
\end{array}\right)+\left(\begin{array}{cc}
\cos \left(\theta_{D E}\right) & -\sin \left(\theta_{D E}\right) \\
\sin \left(\theta_{D E}\right) & \cos \left(\theta_{D E}\right)
\end{array}\right)\left(\begin{array}{l}
\xi \\
\eta
\end{array}\right)
$$




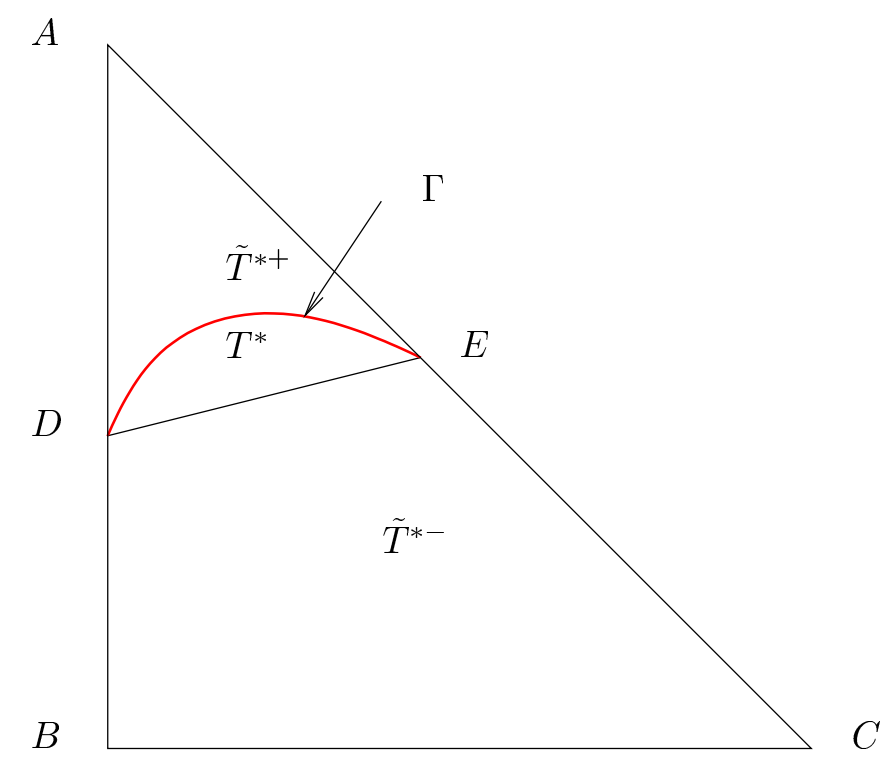

Figure 3: An interface triangle with no obscure point.

where $\left(x_{D}, y_{D}\right)^{T}$ is the coordinates of point $D$ and $\theta_{D E}$ is the angle between $\overline{D E}$ and the $x$ axis. As in [5], we can assume that $\Gamma$ has the following equation in this local system:

$$
\eta=\phi(\xi), \quad \xi \in[0,|\overline{D E}|]
$$

with

$$
\begin{aligned}
& |\phi(\xi)| \leq C h^{2}, \\
& \left|\phi^{\prime}(\xi)\right| \leq C h .
\end{aligned}
$$

¿From now on, if necessary, for any point $P$, we will use

$$
\left(\begin{array}{l}
x_{P} \\
y_{P}
\end{array}\right) \text { and }\left(\begin{array}{l}
\xi_{P} \\
\eta_{P}
\end{array}\right)
$$

to denote its coordinates in the $x-y$ and $\xi-\eta$ systems, respectively.

Lemma 3.1 There exists $h_{0}>0$ such that for all $0 \leq h \leq h_{0}$ and any point $\widetilde{A} \in \Gamma$, we have

$$
\begin{aligned}
& \left\|\widetilde{A}-\widetilde{A}_{\perp}\right\| \leq C h^{2} . \\
& \left\|N \frac{s}{D E}-N^{s}(\widetilde{A})\right\| \leq C h, \quad s=-,+,
\end{aligned}
$$

Proof. We can just prove these in the local coordinate system because the transformation (3.17) preserve the vector length. In the local system, we have

$$
\widetilde{A}=\left(\begin{array}{c}
\tilde{\xi} \\
\phi(\tilde{\xi})
\end{array}\right), \widetilde{A}_{\perp}=\left(\begin{array}{c}
\tilde{\xi} \\
0
\end{array}\right) .
$$

Hence (3.21) is just the consequence of (3.19). Also, we have

$$
\mathbf{n}(\overline{D E})=\left(\begin{array}{c}
0 \\
1
\end{array}\right), \mathbf{n}(\widetilde{A})=\frac{1}{\sqrt{1+\left(\phi^{\prime}(\tilde{\xi})\right)^{2}}}\left(\begin{array}{c}
-\phi^{\prime}(\tilde{\xi}) \\
1
\end{array}\right) .
$$


Then, by (3.20), we have

$$
\|\mathbf{n}(\overline{D E})-\mathbf{n}(\widetilde{A})\| \leq C h
$$

which together with definition of $N_{\overline{D E}}^{s}$ and $N^{s}(\widetilde{A}), s=-,+$, lead to $(3.22)$.

We will call a point $X=(x, y)^{T}$ in an interface triangle $T \in \mathcal{T}_{h}$ an obscure point if one of the three line segments passing $X$ and the vertices of $T$ intersects the interface more than once. Without loss of generality, we discuss an interface triangle not consisting of any obscure point; the arguments used below can be readily extended to handle interface triangles with obscure points.

For any function $u \in P H_{i n t}^{2}(T)$, the error estimates for $I_{h, T} u$ is obtained by estimates over the three subsets $T^{*-}, T^{*+}$ and $T^{*}$ of $T$. The key issue is to establish suitable multipoint Taylor expansions for functions in $S_{h}(T)$ and $P C^{3}(T)$.

We start with the estimation on $T^{*-}$. Let $X=(x, y)^{T}$ be a point in $T^{*-}$. Without loss of generality, we can assume that line segments $\overline{X B}$ and $\overline{X C}$ do not intersect with the interface and $\overline{D E}$, while line segment $\overline{X A}$ meets $\Gamma$ and $\overline{D E}$ at $\widetilde{A}$ and $\bar{A}$, respectively, see Figure 4. Also, we assume that

$$
\begin{aligned}
& \widetilde{A}=\tilde{t} A+(1-\tilde{t}) X=(\widetilde{x}, \widetilde{y})^{T} \\
& \bar{A}=\bar{t} A+(1-\bar{t}) X=(\bar{x}, \bar{y})^{T}
\end{aligned}
$$

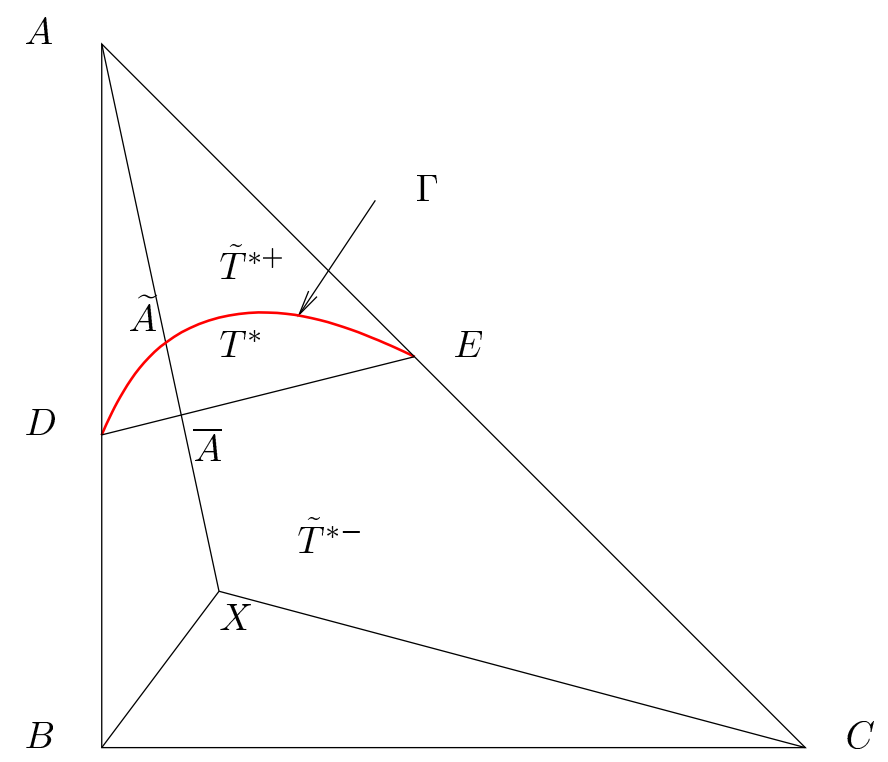

Figure 4: A point $X \in T^{*-}$ is connected to the three vertices by line segments.

Lemma 3.2 Given a real number $r$, a two dimensional vector $\mathbf{q}$, a point $X \in T^{*-}$, and a point $X_{\overline{D E}} \in \overline{D E}$, there exits a function $v \in S_{h}(T)$ such that $v(X)=r, \nabla v(X)=\mathbf{q}$, and

$$
\begin{aligned}
0=\mathbf{q} & \cdot\left((A-X) \phi_{1}(X)+(B-X) \phi_{2}(X)+(C-X) \phi_{3}(X)\right) \\
& +\left(N_{\overline{D E}}^{-}-I\right) \mathbf{q} \cdot(A-X)(1-\widetilde{t}) \phi_{1}(X)+\left(N_{\overline{D E}}^{-}-I\right) \mathbf{q} \cdot\left(\widetilde{A}-X_{\overline{D E}}\right) \phi_{1}(X)
\end{aligned}
$$


Proof. Let

$$
v(Y)= \begin{cases}v^{-}(Y), & Y \in T^{-} \\ v^{+}(Y), & Y \in T^{+}\end{cases}
$$

be a function in $S_{h}(T)$. Since $v(Y)$ is piecewise linear, $v(X)=r, \nabla v(X)=\mathbf{q}$ uniquely determine $v^{-}(Y)$. Then the interface conditions $v^{-}(D)=v^{+}(D), v^{-}(E)=v^{+}(E)$, and $\beta^{-} \frac{\partial v^{-}}{\partial \mathbf{n}_{\overline{D E}}}=$ $\beta^{+} \frac{\partial v^{+}}{\partial \mathbf{n}_{\overline{D E}}}$ uniquely determine $v^{+}(Y)$.

Since $v(t B+(1-t) X)$ is a linear function of $t$, using integration by parts, we have

$$
\begin{aligned}
v(B) & =v(X)+\int_{0}^{1} \frac{d u(t B+(1-t) X)}{d t} d t \\
& =v(X)+\nabla v(X) \cdot(B-X)+\int_{0}^{1}(1-t) \frac{d^{2} u(t B+(1-t) X)}{d t^{2}} d t \\
& =r+\mathbf{q} \cdot(B-X) .
\end{aligned}
$$

Similarly, we have

$$
v(C)=r+\mathbf{q} \cdot(C-X)
$$

For point $A$, we first have

$$
\begin{aligned}
v(A)=v(X) & +v^{+}(\widetilde{A})-v^{-}(\widetilde{A})+\int_{0}^{\bar{t}} \frac{\partial v}{\partial t}(t A+(1-t) X) d t \\
& +\int_{\bar{t}}^{\widetilde{t}} \frac{\partial v}{\partial t}(t A+(1-t) X) d t+\int_{\widetilde{t}}^{1} \frac{\partial v}{\partial t}(t A+(1-t) X) d t
\end{aligned}
$$

Using integration by parts, we have

$$
\begin{aligned}
& \int_{0}^{\bar{t}} \frac{\partial v}{\partial t}(t A+(1-t) X) d t+\int_{\bar{t}}^{\widetilde{t}} \frac{\partial v}{\partial t}(t A+(1-t) X) d t+\int_{\widetilde{t}}^{1} \frac{\partial v}{\partial t}(t A+(1-t) X) d t \\
= & \nabla v(X) \cdot(A-X)-\nabla v(\bar{A}) \cdot(A-x)(1-\bar{t})+\int_{0}^{\bar{t}}(1-t) \frac{\partial^{2} v}{\partial t^{2}}(t A+(1-t) X) d t \\
& +\nabla v(\bar{A}) \cdot(A-X)(1-\bar{t})-\nabla v^{-}(\widetilde{A}) \cdot(A-X)(1-\widetilde{t})+\int_{\bar{t}}^{\widetilde{t}}(1-t) \frac{\partial^{2} v}{\partial t^{2}}(t A+(1-t) X) d t \\
& +\nabla v^{+}(\widetilde{A}) \cdot(A-X)(1-\widetilde{t})+\int_{\widetilde{t}}^{1}(1-t) \frac{\partial^{2} v}{\partial t^{2}}(t A+(1-t) X) d t \\
= & \nabla v(X) \cdot(A-X)-\nabla v^{-}(\widetilde{A}) \cdot(A-X)(1-\widetilde{t})+\nabla v^{+}(\widetilde{A}) \cdot(A-X)(1-\widetilde{t}) \\
= & \nabla v(X) \cdot(A-X)+\left(N_{\overline{D E}}^{-}-I\right) \nabla v^{-}(\widetilde{A}) \cdot(A-X)(1-\widetilde{t}) \\
= & \mathbf{q} \cdot(A-X)+\left(N_{\overline{D E}}^{-}-I\right) \mathbf{q} \cdot(A-X)(1-\widetilde{t}),
\end{aligned}
$$

where all integral terms involving second order derivatives of $v(Y)$ disappear because $v(Y)$ is a piecewise polynomial of degree 1 .

By Taylor expansion, we have

$$
\begin{aligned}
& v^{+}(\widetilde{A})=v^{+}\left(X_{\overline{D E}}\right)+\nabla v^{+}\left(X_{\overline{D E}}\right) \cdot\left(\widetilde{A}-X_{\overline{D E}}\right) \\
& v^{-}(\widetilde{A})=v^{-}\left(X_{\overline{D E}}\right)+\nabla v^{-}\left(X_{\overline{D E}}\right) \cdot\left(\widetilde{A}-X_{\overline{D E}}\right)
\end{aligned}
$$


Hence, from the continuity of $v(Y)$ on $\overline{D E}$,

$$
\begin{aligned}
& v^{+}(\widetilde{A})-v^{-}(\widetilde{A}) \\
= & \left(N_{\overline{D E}}^{-}-I\right) \nabla v^{-}\left(X_{\overline{D E}}\right) \cdot\left(\widetilde{A}-X_{\overline{D E}}\right) \\
= & \left(N_{\overline{D E}}^{-}-I\right) \nabla v(X) \cdot\left(\widetilde{A}-X_{\overline{D E}}\right)=\left(N_{\overline{D E}}^{-}-I\right) \mathbf{q} \cdot\left(\widetilde{A}-X_{\overline{D E}}\right)
\end{aligned}
$$

Putting these together, we have

$$
\begin{aligned}
v(A)=r+ & \mathbf{q} \cdot(A-X)+\left(N_{\overline{D E}}^{-}-I\right) \mathbf{q} \cdot(A-X)(1-\widetilde{t}) \\
& +\left(N_{\overline{D E}}^{-}-I\right) \mathbf{q} \cdot\left(\widetilde{A}-X_{\overline{D E}}\right) .
\end{aligned}
$$

Then, from these expansions of $v(Y)$ at the vertices of $T$, we have

$$
\begin{aligned}
v(X)= & I_{h, T} v(X)=v(A) \phi_{1}(X)+v(B) \phi_{2}(X)+v(C) \phi_{3}(X) \\
= & r \sum_{i=1}^{3} \phi_{i}(X)+\mathbf{q} \cdot\left((A-X) \phi_{1}(X)+(B-X) \phi_{2}(X)+(C-X) \phi_{3}(X)\right) \\
& \quad+\left(N_{\overline{D E}}^{-}-I\right) \mathbf{q} \cdot(A-X)(1-\widetilde{t}) \phi_{1}(X)+\left(N_{\overline{D E}}^{-}-I\right) \mathbf{q} \cdot\left(\widetilde{A}-X_{\overline{D E}}\right) \phi_{1}(X),
\end{aligned}
$$

and the proof is finished because $v(X)=r$ and $\sum_{i=1}^{3} \phi_{i}(X)=1$.

Lemma 3.3 For any $u \in P C^{2}(T), X \in T^{*-}$, and $X_{\overline{D E}} \in \overline{D E}$ we have

$$
\begin{aligned}
& I_{h, T} u(X)-u(X) \\
= & \left(N^{-}(\widetilde{A})-N_{\overline{D E}}^{-}\right) \nabla u(X) \cdot(A-X) \phi_{1}(X)(1-\widetilde{t})-\left(N_{\overline{D E}}^{-}-I\right) \nabla u(X) \cdot\left(\widetilde{A}-X_{\overline{D E}}\right) \phi_{1}(X) \\
& +(1-\tilde{t})\left(N^{-}(\widetilde{A})-I\right) \int_{0}^{1} \frac{d \nabla u^{-}}{d t}(t \widetilde{A}+(1-t) X) \cdot(A-X) d t \phi_{1}(X) \\
& +\int_{0}^{\tilde{t}}(1-t) \frac{d^{2} u}{d t^{2}}(t A+(1-t) X) d t \phi_{1}(X)+\int_{\tilde{t}}^{1}(1-t) \frac{d^{2} u}{d t^{2}}(t A+(1-t) X) d t \phi_{1}(X)(3.24) \\
& +\int_{0}^{1}(1-t) \frac{d^{2} u}{d t^{2}}(t B+(1-t) X) d t \phi_{2}(X)+\int_{0}^{1}(1-t) \frac{d^{2} u}{d t^{2}}(t C+(1-t) X) d t \phi_{3}(X) .
\end{aligned}
$$

Proof. Since $u(t B+(1-t) X)$ is a $C^{2}$ function as a function of $t$, we have

$$
\begin{aligned}
u(B) & =u(X)+\int_{0}^{1} \frac{d u}{d t}(t B+(1-t) X) d t \\
& =u(X)+\nabla u(X) \cdot(B-X)+\int_{0}^{1}(1-t) \frac{d^{2} u}{d t^{2}}(t B+(1-t) X) d t .
\end{aligned}
$$

Similarly, we have

$$
\begin{aligned}
u(C) & =u(X)+\int_{0}^{1} \frac{d u}{d t}(t C+(1-t) X) d t \\
& =u(X)+\nabla u(X) \cdot(C-X)+\int_{0}^{1}(1-t) \frac{d^{2} u}{d t^{2}}(t C+(1-t) X) d t .
\end{aligned}
$$

Using the jump condition across the interface, we have

$$
\nabla u^{+}(\widetilde{A})=\left(\begin{array}{cc}
n_{y}(\widetilde{A})^{2}+\rho n_{x}(\widetilde{A})^{2} & (\rho-1) n_{x}(\widetilde{A}) n_{y}(\widetilde{A}) \\
(\rho-1) n_{x}(\widetilde{A}) n_{y}(\widetilde{A}) & n_{x}(\widetilde{A})^{2}+\rho n_{y}(\widetilde{A})^{2}
\end{array}\right) \nabla u^{-}(\widetilde{A})=N^{-}(\widetilde{A}) \nabla u^{-}(\widetilde{A})
$$


Then, we have

$$
\begin{aligned}
u(A)= & u(X)+\int_{0}^{1} \frac{d u}{d t}(t A+(1-t) X) d t \\
= & u(X)+\int_{0}^{\tilde{t}} \frac{d u}{d t}(t A+(1-t) X) d t+\int_{\tilde{t}}^{1} \frac{d u}{d t}(t A+(1-t) X) d t \\
= & u(X)-\nabla u^{-}(\widetilde{A}) \cdot(A-X)(1-\tilde{t})+\nabla u(X) \cdot(A-X)+\int_{0}^{\tilde{t}}(1-t) \frac{d^{2} u}{d t^{2}}(t A+(1-t) X) d t \\
& +\nabla u^{+}(\widetilde{A}) \cdot(A-X)(1-\tilde{t})+\int_{\tilde{t}}^{1}(1-t) \frac{d^{2} u}{d t^{2}}(t A+(1-t) X) d t \\
= & u(X)+\nabla u(X) \cdot(A-X)+\left(N^{-}(\widetilde{A})-I\right) \nabla u^{-}(\widetilde{A}) \cdot(A-X)(1-\tilde{t}) \\
& +\int_{0}^{\tilde{t}}(1-t) \frac{d^{2} u}{d t^{2}}(t A+(1-t) X) d t+\int_{\tilde{t}}^{1}(1-t) \frac{d^{2} u}{d t^{2}}(t A+(1-t) X) d t \\
= & u(X)+\nabla u(X) \cdot(A-X)+\left(N^{-}(\widetilde{A})-I\right) \nabla u(X) \cdot(A-X)(1-\tilde{t}) \\
& +(1-\tilde{t})\left(N^{-}(\widetilde{A})-I\right) \int_{0}^{1} \frac{d \nabla u^{-}}{d t}(t \widetilde{A}+(1-t) X) \cdot(A-x) d t \\
& +\int_{0}^{\tilde{t}}(1-t) \frac{d^{2} u}{d t^{2}}(t A+(1-t) X) d t+\int_{\tilde{t}}^{1}(1-t) \frac{d^{2} u}{d t^{2}}(t A+(1-t) X) d t .
\end{aligned}
$$

Then

$$
\begin{aligned}
I_{h, T} u(X)= & u(A) \phi_{1}(X)+u(B) \phi_{2}(X)+u(C) \phi_{3}(X) \\
= & u(X) \sum_{i=1}^{3} \phi_{i}(X)+\nabla u(X) \cdot\left((A-X) \phi_{1}(X)+(B-X) \phi_{2}(X)+(C-X) \phi_{3}(X)\right) \\
& +\left(N^{-}(\widetilde{A})-I\right) \nabla u^{-}(X) \cdot(A-X)(1-\tilde{t}) \phi_{1}(X) \\
& +(1-\tilde{t})\left(N^{-}(\widetilde{A})-I\right) \int_{0}^{1} \frac{d \nabla u^{-}}{d t}(t \tilde{A}+(1-t) X) \cdot(A-X) d t \phi_{1}(X) \\
& +\int_{0}^{\tilde{t}}(1-t) \frac{d^{2} u}{d t^{2}}(t A+(1-t) X) d t \phi_{1}(X)+\int_{\tilde{t}}^{1}(1-t) \frac{d^{2} u}{d t^{2}}(t A+(1-t) X) d t \phi_{1}(X) \\
& +\int_{0}^{1}(1-t) \frac{d^{2} u}{d t^{2}}(t B+(1-t) X) d t \phi_{2}(X)+\int_{0}^{1}(1-t) \frac{d^{2} u}{d t^{2}}(t C+(1-t) X) d t \phi_{3}(X) .
\end{aligned}
$$

Now let $v \in S_{h}(T)$ be such that

$$
v(Y)= \begin{cases}v^{+}(Y), & Y \in T^{+} \\ v^{-}(Y), & Y \in T^{-}\end{cases}
$$

with first degree polynomials $v^{-}(Y), v^{+}(Y)$ determined by

$$
\left\{\begin{array}{l}
v^{-}(X)=u^{-}(X), \quad \nabla v^{-}(X)=\nabla u^{-}(X), \\
v^{+}(D)=v^{-}(D), \quad v^{+}(E)=v^{-}(E), \quad \beta^{+} \frac{\partial v^{+}(\bar{A})}{\partial \mathbf{n}(\overline{D E})}=\beta^{-} \frac{\partial v^{-}(\bar{A})}{\partial \mathbf{n}(\overline{D E})}
\end{array}\right.
$$

Then by Lemma 3.2, we have

$$
\begin{aligned}
u(X)= & u(X) \sum_{i=1}^{3} \phi_{i}(X)+\nabla u(X) \cdot\left((A-X) \phi_{1}(X)+(B-X) \phi_{2}(X)+(C-X) \phi_{3}(X)\right) \\
+ & \left(N_{\overline{D E}}^{-}-I\right) \nabla u(X) \cdot(A-X)(1-\widetilde{t}) \phi_{1}(X) \\
& +\left(N_{\overline{D E}}^{-}-I\right) \nabla u(X) \cdot\left(\widetilde{A}-X_{\overline{D E}}\right) \phi_{1}(X)
\end{aligned}
$$


Finally, (3.24) follows from (3.28) and (3.30).

Lemma 3.4 There exits a constant $C$ such that

$$
\left\|I_{h, T} u-u\right\|_{0, T^{*-}} \leq C h^{2}\|u\|_{2, T}
$$

for any $u \in P H_{\text {int }}^{2}(T)$ where $T$ is an arbitrary interface triangle.

Proof. Because of $\left(H_{5}\right)$, we just need to show that (3.31) is true for any $u \in P C^{2}(T)$.

We proceed by estimating the $L^{2}$ norms for all the terms on the right hand side of (3.24). By Lemmas 3.1 and 2.4, we have the following estimate for the $L^{2}$ norms of the first two terms by letting $X_{\overline{D E}}=\widetilde{A}_{\perp}$ :

$$
\begin{aligned}
& \left\|Q_{1}\right\|_{0, T^{*-}}+\left\|Q_{2}\right\|_{0, T^{*-}} \\
= & \left\|\left(N^{-}(\widetilde{A})-N_{\overline{D E}}^{-}\right) \nabla u(X) \cdot(A-X) \phi_{1}(X)(1-\widetilde{t})\right\|_{0, T^{*-}} \\
& +\left\|\left(N_{\overline{D E}}^{-}-I\right) \nabla u^{-}(X) \cdot\left(\widetilde{A}-\widetilde{A}_{\perp}\right) \phi_{1}(X)\right\|_{0, T^{*-}} \\
\leq & C h^{2}\|u\|_{1, T^{*-}} \leq C h^{2}\|u\|_{2, T^{*-}}
\end{aligned}
$$

For the third term, we note that

$$
\begin{aligned}
Q_{3}^{2} & \leq C(1-\widetilde{t})^{2}\left(\int_{0}^{1}\left[u_{x x}(\xi, \eta)(\widetilde{x}-x)^{2}+2 u_{x y}(\xi, \eta)(\widetilde{x}-x)(\widetilde{y}-y)+u_{y y}(\xi, \eta)(\widetilde{y}-y)^{2}\right] d t\right)^{2} \\
& \leq C h^{4}(1-\widetilde{t})^{2}\left(\int_{0}^{1}\left[u_{x x}(\xi, \eta)+2 u_{x y}(\xi, \eta)+u_{y y}(\xi, \eta)\right] d t\right)^{2} \\
& \leq C h^{4}(1-\widetilde{t})^{2} \int_{0}^{1}\left[u_{x x}^{2}(\xi, \eta)+u_{x y}^{2}(\xi, \eta)+u_{y y}^{2}(\xi, \eta)\right] d t
\end{aligned}
$$

with $\xi=t \widetilde{x}+(1-t) x, \eta=t \widetilde{y}+(1-t) y$. Therefore,

$$
\begin{aligned}
\left\|Q_{3}\right\|_{0, T^{*-}}^{2} & =\int_{T^{*-}} Q_{3}^{2} d X \\
& \leq C h^{4}(1-\widetilde{t})^{2} \int_{T^{*-}} \int_{0}^{1}\left[u_{x x}^{2}(\xi, \eta)+u_{x y}^{2}(\xi, \eta)+u_{y y}^{2}(\xi, \eta)\right] d t d X \\
& \leq C h^{4} \int_{T^{-}}\left[u_{x x}^{2}(\xi, \eta)+u_{x y}^{2}(\xi, \eta)+u_{y y}^{2}(\xi, \eta)\right] d X \\
& \leq C h^{4}\|u\|_{2, T^{-}}^{2},
\end{aligned}
$$

or

$$
\left\|Q_{3}\right\|_{0, T^{*-}} \leq C h^{2}\|u\|_{2, T^{-}} .
$$

For the fourth term, we have

$$
\begin{aligned}
Q_{4}^{2} & \leq C\left(\int_{0}^{\widetilde{t}}(1-t)\left[u_{x x}(\xi, \eta)\left(x_{A}-x\right)^{2}+2 u_{x y}(\xi, \eta)\left(x_{A}-x\right)\left(y_{A}-y\right)+u_{y y}(\xi, \eta)\left(y_{A}-y\right)^{2}\right] d t\right)^{2} \\
& \leq C h^{4}\left(\int_{0}^{\widetilde{t}}(1-t)\left[u_{x x}(\xi, \eta)+2 u_{x y}(\xi, \eta)+u_{y y}(\xi, \eta)\right] d t\right)^{2} \\
& \leq C h^{4} \int_{0}^{\widetilde{t}}(1-t)^{2}\left[u_{x x}^{2}(\xi, \eta)+u_{x y}^{2}(\xi, \eta)+u_{y y}^{2}(\xi, \eta)\right] d t
\end{aligned}
$$


with $\xi=t x_{A}+(1-t) x, \eta=t y_{A}+(1-t) y, A=\left(x_{A}, y_{A}\right)^{T}$. Therefore

$$
\begin{aligned}
\left\|Q_{4}\right\|_{T^{*-}}^{2} & \leq C h^{4} \int_{T^{*-}} \int_{0}^{\widetilde{t}}(1-t)^{2}\left[u_{x x}^{2}(\xi, \eta)+u_{x y}^{2}(\xi, \eta)+u_{y y}^{2}(\xi, \eta)\right] d t d X \\
& \leq C h^{4} \int_{T^{-}}\left[u_{x x}^{2}(\xi, \eta)+u_{x y}^{2}(\xi, \eta)+u_{y y}^{2}(\xi, \eta)\right] d X \leq C h^{4}\|u\|_{2, T^{-}}
\end{aligned}
$$

or

$$
\left\|Q_{4}\right\|_{T^{*-}} \leq C h^{2}\|u\|_{2, T^{-}} .
$$

Similarly, we can show that

$$
\begin{aligned}
\left\|Q_{5}\right\|_{T^{*-}} & \leq C h^{2}\|u\|_{2, T^{+}}, \\
\left\|Q_{6}\right\|_{T^{*-}} & \leq C h^{2}\|u\|_{2, T^{-}}, \\
\left\|Q_{7}\right\|_{T^{*-}} & \leq C h^{2}\|u\|_{2, T^{-}} .
\end{aligned}
$$

Finally, (3.31) follows from the estimates for $Q_{i}, i=1,2, \cdots, 7$ above.

We now turn to the estimation in $H^{1}$ norm on $T^{*-}$. In the following two lemmas, we let $I_{1}, I_{2}$ and $I_{3}$ be the integral terms in the expansions (3.27), (3.25), and (3.26), respectively.

Lemma 3.5 For any $u \in P C^{3}(T), X \in T^{*-}$, and $X_{\overline{D E}} \in \overline{D E}$ we have

$$
\begin{aligned}
& \frac{\partial\left(I_{h, T} u(X)-u(X)\right)}{\partial s}=\left(N^{-}(\widetilde{A})-N_{\overline{D E}}^{-}\right) \nabla u(X)(A-\widetilde{A}) \frac{\partial \phi_{1}(X)}{\partial s} \\
& -\left(N_{\overline{D E}}^{-}-I\right) \nabla u(X)\left(\widetilde{A}-X_{\overline{D E}}\right) \frac{\partial \phi_{1}(X)}{\partial s}+\left(I_{1} \frac{\partial \phi_{1}}{\partial s}+I_{2} \frac{\partial \phi_{2}}{\partial s}+I_{3} \frac{\partial \phi_{3}}{\partial s}\right), s=x, y .
\end{aligned}
$$

Proof. We give a proof only for the case in which $s=x$ because the case in which $s=y$ can be carried out similarly. From (3.24) in Lemma 3.3, we have

$$
\begin{aligned}
\frac{\partial\left(I_{h, T} u(X)-u(X)\right)}{\partial x}= & \frac{\partial}{\partial x}\left[\left(N^{-}(\widetilde{A})-N_{\overline{D E}}^{-}\right) \nabla u(X)(A-\widetilde{A})\right] \phi_{1}(X) \\
& +\left(N^{-}(\widetilde{A})-N_{\overline{D E}}^{-}\right) \nabla u(X)(A-\widetilde{A}) \frac{\partial \phi_{1}(X)}{\partial x} \\
& -\frac{\partial}{\partial x}\left[\left(N_{\overline{D E}}^{-}-I\right) \nabla u(X)\left(\widetilde{A}-X_{\overline{D E}}\right)\right] \phi_{1}(X) \\
& -\left(N_{\overline{D E}}^{-}-I\right) \nabla u(X)\left(\widetilde{A}-X_{\overline{D E}}\right) \frac{\partial \phi_{1}(X)}{\partial x} \\
& +\left(\frac{\partial I_{1}}{\partial x} \phi_{1}(X)+\frac{\partial I_{2}}{\partial x} \phi_{2}(X)+\frac{\partial I_{3}}{\partial x} \phi_{3}(X)\right)+\left(I_{1} \frac{\partial \phi_{1}}{\partial x}+I_{2} \frac{\partial \phi_{2}}{\partial x}+I_{3} \frac{\partial \phi_{3}}{\partial x}\right) .
\end{aligned}
$$

¿From the expansions (3.27), (3.25), and (3.26), we have

$$
\begin{aligned}
0= & \frac{\partial^{2} u(X)}{\partial x^{2}}\left(x_{A}-x\right)+\frac{\partial^{2} u(X)}{\partial x \partial y}\left(y_{A}-y\right) \\
& \quad+\frac{\partial}{\partial x}\left[\left(N^{-}(\widetilde{A})-I\right) \nabla u(X)(A-\widetilde{A})\right]+\frac{\partial I_{1}}{\partial x}, \\
0= & \frac{\partial^{2} u(X)}{\partial x^{2}}\left(x_{B}-x\right)+\frac{\partial^{2} u(X)}{\partial x \partial y}\left(y_{B}-y\right)+\frac{\partial I_{2}}{\partial x}, \\
0= & \frac{\partial^{2} u(X)}{\partial x^{2}}\left(x_{C}-x\right)+\frac{\partial^{2} u(X)}{\partial x \partial y}\left(y_{C}-y\right)+\frac{\partial I_{3}}{\partial x} .
\end{aligned}
$$


Then

$$
\begin{gathered}
\frac{\partial I_{1}}{\partial x} \phi_{1}(X)+\frac{\partial I_{2}}{\partial x} \phi_{2}(X)+\frac{\partial I_{3}}{\partial x} \phi_{3}(X) \\
=-\left[\mathbf{p} \cdot(A-X) \phi_{1}(X)+\mathbf{p} \cdot(B-X) \phi_{2}(X)+\mathbf{p} \cdot(C-X) \phi_{3}(X)\right] \\
-\frac{\partial}{\partial x}\left[\left(N^{-}(\widetilde{A})-I\right) \nabla u(X)(A-\widetilde{A})\right] \phi_{1}(X),
\end{gathered}
$$

where

$$
\mathbf{p}=\left(\begin{array}{c}
\frac{\partial^{2} u(X)}{\partial x^{2}} \\
\frac{\partial^{2} u(X)}{\partial x \partial y}
\end{array}\right)
$$

Let $v(Y) \in S_{h}$ be such that $\nabla v(X)=\mathbf{p}$. Then by Lemma 3.2, we have

$$
\begin{aligned}
0=\mathbf{p} & \cdot(A-X) \phi_{1}(x)+\mathbf{p} \cdot(B-X) \phi_{2}(x)+\mathbf{p} \cdot(C-X) \phi_{3}(x) \\
+ & \left(N_{\overline{D E}}^{-}-I\right) \mathbf{p} \cdot(A-\widetilde{A}) \phi_{1}(X)+\left(N_{\overline{D E}}^{-}-I\right) \mathbf{p} \cdot\left(\widetilde{A}-X_{\overline{D E}}\right) \phi_{1}(X) .
\end{aligned}
$$

Hence

$$
\begin{aligned}
& \frac{\partial I_{1}}{\partial x} \phi_{1}(X)+\frac{\partial I_{2}}{\partial x} \phi_{2}(X)+\frac{\partial I_{3}}{\partial x} \phi_{3}(X) \\
= & \left(N_{\overline{D E}}^{-}-I\right) \mathbf{p} \cdot(A-\widetilde{A}) \phi_{1}(X)+\left(N_{\overline{D E}}^{-}-I\right) \mathbf{p} \cdot\left(\widetilde{A}-X_{\overline{D E}}\right) \phi_{1}(X) \\
& -\frac{\partial}{\partial x}\left[\left(N^{-}(\widetilde{A})-I\right) \nabla u(X)(\widetilde{A}-X)\right] \phi_{1}(X),
\end{aligned}
$$

and

$$
\begin{aligned}
& \frac{\partial\left(I_{h, T} u(X)-u(X)\right)=}{\partial x}=\frac{\partial}{\partial x}\left[\left(N^{-}(\widetilde{A})-N_{\overline{D E}}^{-}\right) \nabla u(X)(A-\widetilde{A})\right] \phi_{1}(X) \\
&+\left(N^{-}(\widetilde{A})-N_{\overline{D E}}^{-}\right) \nabla u(X)(A-\widetilde{A}) \frac{\partial \phi_{1}(X)}{\partial x} \\
&-\frac{\partial}{\partial x}\left[\left(N_{\overline{D E}}^{-}-I\right) \nabla u(X)\left(\widetilde{A}-X_{\overline{D E}}\right)\right] \phi_{1}(X) \\
&-\left(N_{\overline{D E}}^{-}-I\right) \nabla u(X)\left(\widetilde{A}-X_{\overline{D E}}\right) \frac{\partial \phi_{1}(X)}{\partial x} \\
&+\left(N_{\overline{D E}}^{-}-I\right) \mathbf{p} \cdot(A-\widetilde{A}) \phi_{1}(X)+\left(N_{\overline{D E}}^{-}-I\right) \mathbf{p} \cdot\left(\widetilde{A}-X_{\overline{D E}}\right) \phi_{1}(X) \\
&-\frac{\partial}{\partial x}\left[\left(N^{-}(\widetilde{A})-I\right) \nabla u(X)(A-\widetilde{A})\right] \phi_{1}(X) \\
&+\left(I_{1} \frac{\partial \phi_{1}}{\partial x}+I_{2} \frac{\partial \phi_{2}}{\partial x}+I_{3} \frac{\partial \phi_{3}}{\partial x}\right) \\
&=\left.N^{-}(\widetilde{A})-N_{\overline{D E}}^{-}\right) \nabla u(X)(A-\widetilde{A}) \frac{\partial \phi_{1}(X)}{\partial x} \\
&-\left(N_{\overline{D E}}^{-}-I\right) \nabla u(X)\left(\widetilde{A}-X_{\overline{D E}}\right) \frac{\partial \phi_{1}(X)}{\partial x} \\
&+\left(N_{\overline{D E}}^{-}-I\right) \mathbf{p} \cdot(A-\widetilde{A}) \phi_{1}(X)+\left(N_{\overline{D E}}^{-}-I\right) \mathbf{p} \cdot\left(\widetilde{A}-X_{\overline{D E}}\right) \phi_{1}(X) \\
&+\left(I_{1} \frac{\partial \phi_{1}}{\partial x}+I_{2} \frac{\partial \phi_{2}}{\partial x}+I_{3} \frac{\partial \phi_{3}}{\partial x}\right)+\frac{\partial}{\partial x}\left[\left(I-N_{\overline{D E}}^{-}\right) \nabla u(X)\left(A-X_{\overline{D E}}\right)\right] \phi_{1}(X) \\
&=\left(N^{-}(\widetilde{A})-N_{\overline{D E}}^{-}\right) \nabla u(X)(A-\widetilde{A}) \frac{\partial \phi_{1}(X)}{\partial x} \\
&-\left(N_{\overline{D E}}^{-}-I\right) \nabla u(X)\left(\widetilde{A}-X_{\overline{D E}}\right) \frac{\partial \phi_{1}(X)}{\partial x}+\left(I_{1} \frac{\partial \phi_{1}}{\partial x}+I_{2} \frac{\partial \phi_{2}}{\partial x}+I_{3} \frac{\partial \phi_{3}}{\partial x}\right)
\end{aligned}
$$


Lemma 3.6 There exits a constant $C$ such that

$$
\left\|\frac{\partial\left(I_{h, T} u-u\right)}{\partial s}\right\|_{0, T^{*-}} \leq C h\|u\|_{2, T}, \quad s=x, y,
$$

for any $u \in P H^{2}(T)$ where $T$ is an arbitrary interface triangle.

Proof. Because of $\left(H_{5}\right)$, we just need to show that $(3.34)$ is true for any $u \in P C^{3}(T)$. The result follows by letting $X_{\overline{D E}}=\widetilde{A}_{\perp}$ in (3.32) and applying arguments similar to those used in the proof of Lemma 3.4. Note that (2.13) in Lemma 2.4 has to be used here.

The estimation on $T^{*+}$ is rather similar. We state the results in the following four lemmas. Please see see Figure 5 for the notations involved. In particular, we let

$$
\begin{aligned}
& \widetilde{B}=\tilde{t}_{B} B+\left(1-\tilde{t}_{B}\right) X=\left(\widetilde{x}_{B}, \widetilde{y}_{B}\right)^{T}, \\
& \bar{B}=\bar{t}_{B} B+\left(1-\bar{t}_{B}\right) X=\left(\bar{x}_{B}, \bar{y}_{B}\right)^{T}, \\
& \widetilde{C}=\tilde{t}_{C} C+\left(1-\tilde{t}_{C}\right) X=\left(\widetilde{x}_{C}, \widetilde{y}_{C}\right)^{T}, \\
& \bar{C}=\bar{t}_{C} C+\left(1-\bar{t}_{C}\right) X=\left(\bar{x}_{C}, \bar{y}_{C}\right)^{T} .
\end{aligned}
$$

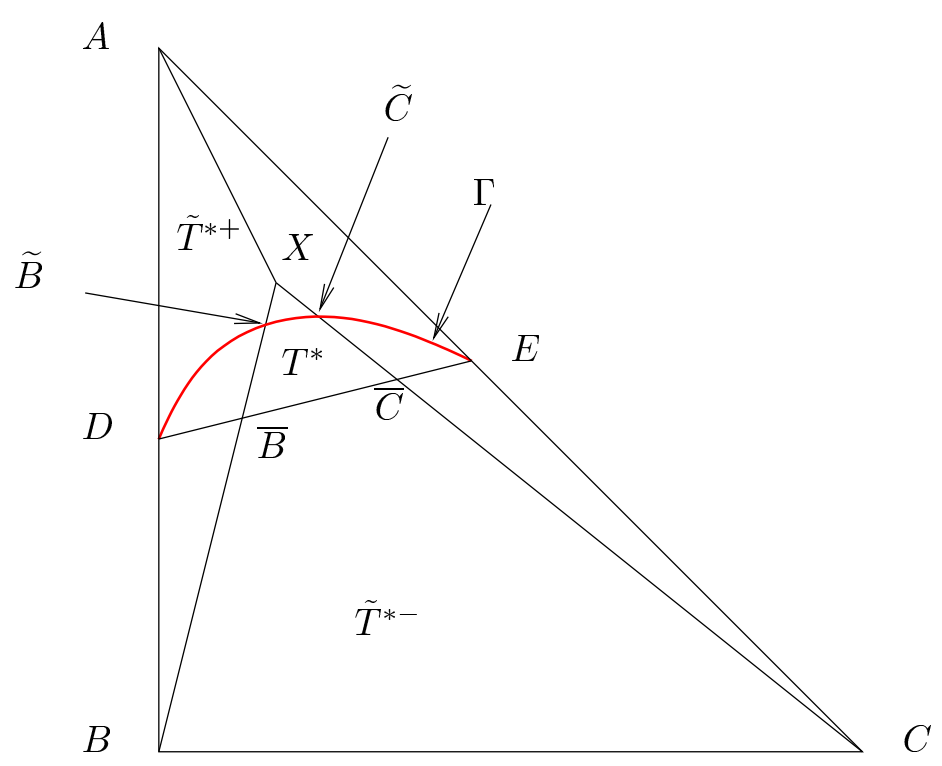

Figure 5: A point $X \in T^{*+}$ is connected to the three vertices by line segments.

Lemma 3.7 Given real number $r$, a two dimensional vector $\mathbf{q}$, a point $X \in T^{*+}$, and two points $X_{\overline{D E}, B} \in \overline{D E}, X_{\overline{D E}, C} \in \overline{D E}$, there exits a function $v \in S_{h}(T)$ such that $v(X)=r, \nabla v(X)=\mathbf{q}$, and

$$
\begin{aligned}
0= & \mathbf{q} \cdot\left((A-X) \phi_{1}(x)+(B-X) \phi_{2}(x)+(C-X) \phi_{3}(x)\right) \\
& +\left(N_{\overline{D E}}^{+}-I\right) \mathbf{q} \cdot\left(\widetilde{B}-X_{\overline{D E}, B}\right) \phi_{2}(X)+\left(N_{\overline{D E}}^{+}-I\right) \mathbf{q}(B-X)\left(1-\widetilde{t}_{B}\right) \phi_{2}(X) \\
& +\left(N_{\overline{D E}}^{+}-I\right) \mathbf{q} \cdot\left(\widetilde{C}-X_{\overline{D E}, C}\right) \phi_{3}(X)+\left(N_{\overline{D E}}^{+}-I\right) \mathbf{q}(C-X)\left(1-\widetilde{t}_{C}\right) \phi_{3}(X) .
\end{aligned}
$$


Lemma 3.8 For any $u \in P C^{2}(T), X \in T^{*+}, X_{\overline{D E}, B} \in \overline{D E}$ and $X_{\overline{D E}, C} \in \overline{D E}$ we have

$$
\begin{aligned}
& I_{h, T} u(X)-u(X) \\
= & \left(N^{+}(\widetilde{B})-N_{\overline{D E}}^{+}\right) \nabla u^{+}(X) \cdot(B-X)\left(1-\widetilde{t}_{B}\right) \phi_{2}(X) \\
& +\left(N^{+}(\widetilde{C})-N_{\overline{D E}}^{+}\right) \nabla u^{+}(X) \cdot(C-X)\left(1-\widetilde{t}_{C}\right) \phi_{3}(X) \\
& -\left(N_{\overline{D E}}^{+}-I\right) \nabla u(X) \cdot\left(\widetilde{B}-B_{\perp}\right) \phi_{2}(X)-\left(N_{\overline{D E}}^{+}-I\right) \nabla u(X) \cdot\left(\widetilde{C}-C_{\perp}\right) \phi_{3}(X) \\
& +\int_{0}^{1}(1-t) \frac{d^{2} u}{d t^{2}}(t A+(1-t) X) d t \phi_{1}(X)+\int_{0}^{\widetilde{t}_{B}}(1-t) \frac{d^{2} u}{d t^{2}}(t B+(1-t) X) d t \phi_{2}(X) \\
& +\int_{\widetilde{t}_{B}}^{1}(1-t) \frac{d^{2} u}{d t^{2}}(t B+(1-t) X) d t \phi_{2}(X) \\
& +\int_{0}^{\widetilde{t}_{C}}(1-t) \frac{d^{2} u}{d t^{2}}(t C+(1-t) X) d t \phi_{3}(X)+\int_{\widetilde{t}_{C}}^{1}(1-t) \frac{d^{2} u}{d t^{2}}(t C+(1-t) X) d t \phi_{3}(X) \\
& +\left(N^{+}(\widetilde{B})-I\right)\left(\int_{0}^{1} \frac{d \nabla u^{+}(t \widetilde{B}+(1-t) X)}{d t} d t\right) \cdot(B-X)\left(1-\widetilde{t}_{B}\right) \phi_{2}(X) \\
& +\left(N^{+}(\widetilde{C})-I\right)\left(\int_{0}^{1} \frac{d \nabla u^{+}(t \widetilde{C}+(1-t) X)}{d t} d t\right) \cdot(C-X)\left(1-\widetilde{t}_{C}\right) \phi_{3}(X) .
\end{aligned}
$$

Lemma 3.9 There exits a constant $C$ such that

$$
\left\|I_{h, T} u-u\right\|_{0, T^{*+}} \leq C h^{2}\|u\|_{2, T},
$$

for any $u \in P H^{2}(T)$ where $T$ is an arbitrary interface triangle.

Lemma 3.10 There exits a constant $C$ such that

$$
\left\|\frac{\partial I_{h, T} u-u}{\partial s}\right\|_{0, T^{*+}} \leq C h\|u\|_{2, T}, \quad s=x, y
$$

for any $u \in P H^{2}(T)$ where $T$ is an arbitrary interface triangle.

Similar multipoint expansions can be established on $T^{*}$. Please see Figure 6 for the notations involved. In particular, we let

$$
\begin{aligned}
& \widetilde{A}=\widetilde{t}_{A} A+\left(1-\widetilde{t}_{A}\right) X=\left(\widetilde{x}_{A}, \widetilde{y}_{A}\right), \\
& \bar{B}=\bar{t}_{B} B+\left(1-\bar{t}_{B}\right) X=\left(\bar{x}_{B}, \bar{y}_{B}\right)^{T}, \\
& \bar{C}=\bar{t}_{C} C+\left(1-\bar{t}_{C}\right) X=\left(\bar{x}_{C}, \bar{y}_{C}\right)^{T} .
\end{aligned}
$$




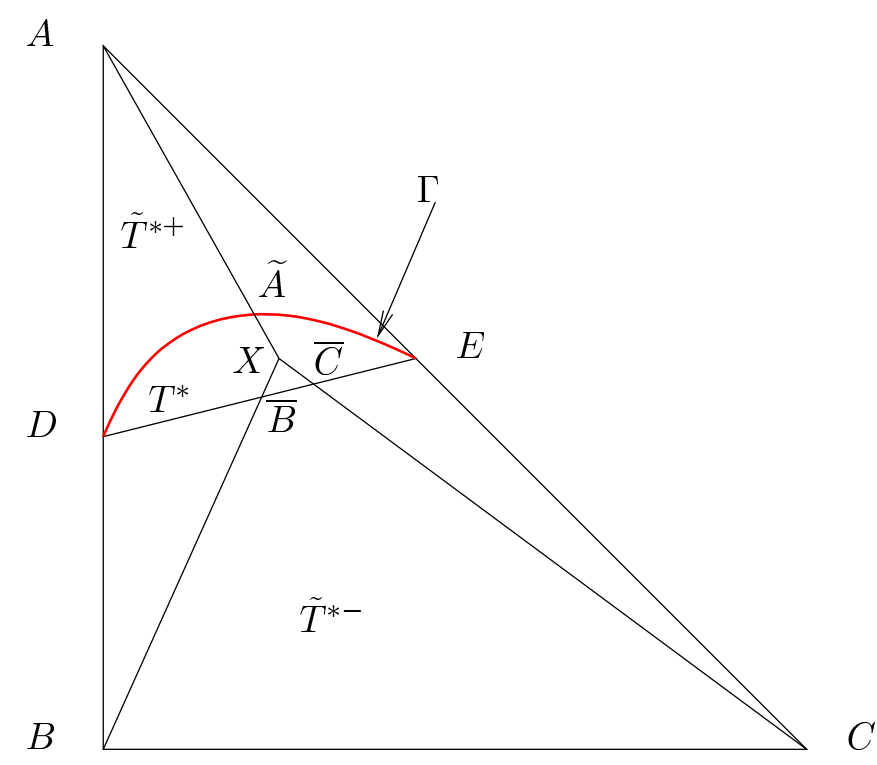

Figure 6: A point $X \in T^{*}$ is connected to the three vertices by line segments.

Lemma 3.11 Given a real number $r$, a two dimensional vector $\mathbf{q}$, a point $X \in T^{*}$, and a point $X_{\overline{D E}} \in \overline{D E}$, there exits a function $v \in S_{h}(T)$ such that $v(X)=r, \nabla v(X)=\mathbf{q}$, and

$$
\begin{aligned}
0= & \mathbf{q} \cdot\left((A-X) \phi_{1}(X)+(B-X) \phi_{2}(X)\left(+(C-X) \phi_{3}(X)\right)\right. \\
& +\left(N_{\overline{D E}}^{+}-I\right) \mathbf{q}\left(\widetilde{A}-X_{\overline{D E}}\right) \phi_{1}(X)+\left(I-N_{\overline{D E}}^{+}\right) \mathbf{q} \cdot(A-X)\left(1-\widetilde{t}_{A}\right) \phi_{1}(X), \\
& +\left(N_{\overline{D E}}^{+}-I\right) \mathbf{q} \cdot(B-X)\left(1-\bar{t}_{B}\right) \phi_{2}(X)+\left(N_{\overline{D E}}^{+}-I\right) \mathbf{q} \cdot(C-X)\left(1-\bar{t}_{C}\right) \phi_{3}(X) .
\end{aligned}
$$

Lemma 3.12 For any $u \in P C^{2}(T)$ we have

$$
\begin{aligned}
& I_{h, T} u(X)-u(X) \\
= & \left(N^{-}(\widetilde{A})-I\right) \nabla u(X) \cdot(A-X)\left(1-\widetilde{t}_{A}\right) \phi_{1}(X) \\
& -\left(I-N_{\overline{D E}}^{+}\right) \nabla u(X) \cdot(A-X)\left(1-\widetilde{t}_{A}\right) \phi_{1}(X), \\
& -\left(N_{\overline{D E}}^{+}-I\right) \nabla u(X) \cdot(B-X)\left(1-\bar{t}_{B}\right) \phi_{2}(X)-\left(N_{\overline{D E}}^{+}-I\right) \nabla u(X) \cdot(C-X)\left(1-\bar{t}_{C}\right) \phi_{3}(X) \\
& -\left(N_{\overline{D E}}^{+}-I\right) \nabla u(X)\left(\widetilde{A}-\widetilde{A}_{\perp}\right) \phi_{1}(X) \\
& +\left(1-\widetilde{t}_{A}\right)\left(N^{-}(\widetilde{A})-I\right) \int_{0}^{1} \frac{d \nabla u^{-}}{d t}(t \widetilde{A}+(1-t) X) \cdot(A-X) d t \phi_{1}(X) \\
& +\int_{0}^{\widetilde{t}_{A}}(1-t) \frac{d^{2} u}{d t^{2}}(t A+(1-t) X) d t \phi_{1}(X)+\int_{\widetilde{t}_{A}}^{1}(1-t) \frac{d^{2} u}{d t^{2}}(t A+(1-t) X) d t \phi_{1}(X) \\
& +\int_{0}^{1}(1-t) \frac{d^{2} u}{d t^{2}}(t B+(1-t) X) d t \phi_{2}(X)+\int_{0}^{1}(1-t) \frac{d^{2} u}{d t^{2}}(t C+(1-t) X) d t \phi_{3}(X) .
\end{aligned}
$$

However, the error estimates on $T^{*}$ is quite different from those obtained on $T^{* s}, s=-,+$. 
Lemma 3.13 There exists a constant $C$ such that

$$
\left\|I_{h, T} u-u\right\|_{0, T^{*}} \leq C h^{2}\|u\|_{2, T}+C h^{\frac{5}{2}-\frac{3}{p}}\|u\|_{1, p, T^{*}},
$$

for any $u \in J(T)$, where $T$ is an arbitrary interface triangle.

Proof. The proof is similar to that for Lemma 3.4 we only need to derive estimates for the first five terms in (3.39). For the first term, we have

$$
\begin{aligned}
\left\|Q_{1}\right\|_{0, T^{*}} & \leq C h\left(\int_{T^{*}}\|\nabla u(X)\|^{2} d X\right)^{1 / 2} \\
& \leq C h\left(\left[\int_{T^{*}} d X\right]^{1 / q}\left[\int_{T^{*}}\|\nabla u(X)\|^{2 p^{\prime}} d X\right]^{1 / p^{\prime}}\right)^{1 / 2}\left(\frac{1}{q}+\frac{1}{p^{\prime}}=1, \quad 1<p^{\prime}, q<\infty\right) \\
& =C h m e s\left(T^{*}\right)^{\frac{1}{2 q}}\left(\int_{T^{*}}\|\nabla u(X)\|^{2 p^{\prime}} d X\right)^{1 /\left(2 p^{\prime}\right)} \\
& \leq C h^{\frac{5}{2}-\frac{3}{p}}\left(\int_{T^{*}}\|\nabla u(X)\|^{p} d X\right)^{1 / p}\left(p=2 p^{\prime}, \operatorname{mes}\left(T^{*}\right) \leq C h^{3}\right) \\
& =C h^{\frac{5}{2}-\frac{3}{p}}\|u\|_{1, p, T^{*}} .
\end{aligned}
$$

Similarly, we can show that

$$
\left\|Q_{i}\right\|_{0, T^{*}} \leq C h^{\frac{5}{2}-\frac{3}{p}}\|u\|_{1, p, T^{*}}, \quad i=2,3, \cdots, 5 .
$$

Putting these together we have (3.40).

Similarly, we have

Lemma 3.14 There exits a constant $C$ such that

$$
\left\|\frac{\partial\left(I_{h, T} u-u\right)}{\partial s}\right\|_{0, T^{*}} \leq C h\|u\|_{2, T}+C h^{\frac{3}{2}-\frac{3}{p}}\|u\|_{1, p, T^{*}}, \quad s=x, y,
$$

for any $u \in P H^{2}(T)$ where $T$ is an arbitrary interface triangle.

We now derive the error estimates for the interpolation $I_{h} u$ in $S_{h}(\Omega)$. Let $\mathcal{T}_{h, \text { int }}$ denote the set of all the interface triangles, and let $\Omega^{\prime}$ be the subset of $\Omega$ formed by the union of all the interface triangles.

Theorem 3.1 There exists a constant $C$ such that

$$
\begin{aligned}
& \left\|I_{h} u-u\right\|_{0, \Omega} \leq C h^{2}|\log (h)|^{1 / 2}\|u\|_{2, \Omega}, \\
& \left\|\frac{\partial\left(I_{h} u-u\right)}{\partial s}\right\|_{0, \Omega} \leq C h|\log (h)|^{1 / 2}\|u\|_{2, \Omega}, s=x, y,
\end{aligned}
$$

for any $u \in P H_{\text {int }}^{2}(\Omega)$ and $h>0$ small enough. 
Proof. From Lemmas 3.4, 3.9, and 3.13, we have

$$
\begin{aligned}
\left\|I_{h} u-u\right\|_{0, \Omega^{\prime}}^{2} & =\sum_{T \in \mathcal{T}_{h, i n t}}\left\|I_{h, T} u-u\right\|_{0, T}^{2} \\
& \leq C h^{4} \sum_{T \in \mathcal{T}_{h, i n t}}\|u\|_{2, T}^{2}+C \sum_{T \in \mathcal{T}_{h, i n t}} h^{5-\frac{6}{p}}\|u\|_{1, p, T^{*}}^{2} \\
& =C h^{4}\|u\|_{2, \Omega^{\prime}}^{2}+C h^{5-\frac{6}{p}} \sum_{T \in \mathcal{T}_{h, i n t}}\|u\|_{1, p, T^{*}}^{2} \\
& \leq C h^{4}\|u\|_{2, \Omega^{\prime}}^{2}+C h^{5-\frac{6}{p}}\left(\sum_{T \in \mathcal{T}_{h, i n t}} 1^{q}\right)^{1 / q}\left(\sum_{T \in \mathcal{T}_{h, i n t}}\|u\|_{1, p, T^{*}}^{p}\right)^{2 / p} .
\end{aligned}
$$

Since the family of partitions $\mathcal{T}_{h}$ is regular, have

$$
\sum_{T \in \mathcal{T}_{h, \text { int }}} 1=C h^{-1} .
$$

Following the same argument used in [4], we also have the following estimate from [8]:

$$
\|u\|_{1, p, \Omega^{\prime}}^{2} \leq C p\|u\|_{2, \Omega^{\prime}}^{2},
$$

where $C$ is a constant independent of $p \in[2, \infty)$. Putting these in the above, and letting $p=|\ln (h)|$ for $h>0$ small enough, we have

$$
\begin{aligned}
\left\|I_{h} u-u\right\|_{0, \Omega^{\prime}}^{2} & \leq C h^{4}\|u\|_{2, \Omega^{\prime}}^{2}+C h^{5-\frac{6}{p}} h^{-\frac{1}{q}}\|u\|_{1, p, \Omega^{\prime}}^{2} \\
& =C h^{4}\|u\|_{2, \Omega^{\prime}}^{2}+C h^{4-\frac{4}{p}}\|u\|_{1, p, \Omega^{\prime}}^{2} \\
& \leq C h^{4}\|u\|_{2, \Omega^{\prime}}^{2}+C h^{4-\frac{4}{p}} p\|u\|_{2, \Omega^{\prime}}^{2} \\
& \leq C h^{4}\|u\|_{2, \Omega^{\prime}}^{2}+C h^{4}|\log (h)|\|u\|_{2, \Omega^{\prime}}^{2} \\
& =C h^{4}(1+|\log (h)|)\|u\|_{2, \Omega^{\prime}}^{2} \\
& =C h^{4}|\log (h)|\|u\|_{2, \Omega^{\prime}}^{2}
\end{aligned}
$$

The estimate (3.41) of this theorem then follows by combining the above estimate and the estimate from the standard finite element interpolation theory:

$$
\left\|I_{h} u-u\right\|_{0, \Omega / \Omega^{\prime}}^{2} \leq C h^{4}\|u\|_{2}^{2} .
$$

Similar derivations can be carried out to obtain (3.42).

\section{Conclusions}

In this paper, we have discussed a nonconforming immersed finite element (IFE) space that can be used to solve interface problems of second order elliptic partial differential equations. The partition of this IFE space is very simple because it can be formed without consideration of the interface location. If applicable, even a Cartesian partition can be used in this IFE space to solve a problem with a rather arbitrary interface. The IFE space is closely related to the standard finite element space formed by piecewise first degree polynomials except for functions over interface triangles. Over an interface triangle, IFE functions are formed according to the 
jump conditions of the itnerface problem to be solved. We have employed the multipoint Taylor expansion technique to analyze the interpolation errors in the IFE space for functions in the Sobolev space related to the interface problems. It has been shown that the IFE space has an approximation capability similar to that of the standard linear finite element space except for a logarithm factor. The estimates for the interpolation error obtained here are critical for deriving error estimates of the finite element (volume) solution to an interface problem based on this IFE space.

\section{References}

[1] D. Braess. Finite Elements. Cambridge University Press, 1997.

[2] S.C. Brenner and L.R. Scott. The Mathematical Theorey of Finite Element Methods. Springer-Verlag, New York, 1994.

[3] C.M. Chen. Some estimates for interpolation approximations and their applications. Numer. Math. J. Chinese Univ., 6(1):35-43, 1984.

[4] Z. Chen and J. Zhou. Finite element methods and their convergence for elliptic and parabolic interface problems. Numer. Math., 79:175-202, 1998.

[5] M. Feistauer and A. Ženišek. Finite element solution of nonlinear elliptic problems. Numer. Math., 50:451-475, 1987.

[6] W. McLean. Strongly Elliptic Systems and Boundary Integral Equations. Cambridge University Press, Cambridge, 2000.

[7] A. Quarteroni and A. Valli. Numerical Approximation of Partial Differential Equations, volume 23 of Computational Mathematics. Springer-Verlag, New York, 2nd edition, 1997.

[8] X. Ren and J. Wei. On a two-dimensional elliptic problem with large exponent in nonlinearity. Trans. Amer. Math. Soc., 343:749-763, 1994.

[9] J. Wloka. Partial Differential Equations. Cambridge University Press, Cambridge, 1987. 